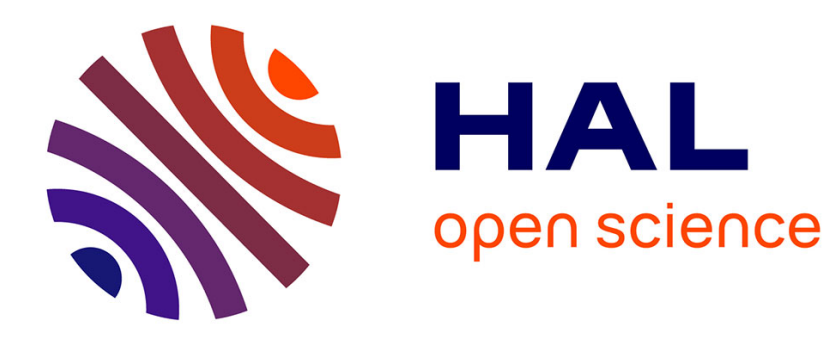

\title{
Welfare, Competition, Specialization and Growth
}

Daria Onori

\section{To cite this version:}

Daria Onori. Welfare, Competition, Specialization and Growth. 2011. halshs-00580722

\section{HAL Id: halshs-00580722 \\ https://shs.hal.science/halshs-00580722}

Preprint submitted on 29 Mar 2011

HAL is a multi-disciplinary open access archive for the deposit and dissemination of scientific research documents, whether they are published or not. The documents may come from teaching and research institutions in France or abroad, or from public or private research centers.
L'archive ouverte pluridisciplinaire HAL, est destinée au dépôt et à la diffusion de documents scientifiques de niveau recherche, publiés ou non, émanant des établissements d'enseignement et de recherche français ou étrangers, des laboratoires publics ou privés. 


\section{GREQAM}

Groupement de Recherche en Economie Quantitative d'Aix-Marseille - UMR-CNRS 6579

Ecole des Hautes études en Sciences Sociales Universités d'Aix-Marseille II et III
Document de Travail n$^{\circ} 2011-16$

\section{Welfare, Competition,}

Specialization and Growth

\section{Daria Onori}

March 2011 


\title{
Welfare, Competition, Specialization and Growth
}

\author{
Daria Onori*
}

March 28, 2011

\begin{abstract}
In this paper we consider a simple model of horizontal differentiation and derive the closed form solutions for the level of the variables in the decentralized economy and in the social planner case. This enables us to analyze consumers' welfare as a function of the parameter representing market power. We surprisingly find that, when the total labor force is greater than a certain level, the welfare function is an inverted-N shape in the decentralized economy and monotonically decreasing in the centralized economy. This suggests that there is another effect which interacts with market power: the degree of returns to specialization.
\end{abstract}

Keywords: Closed form solutions; Welfare; Competition; Degree of returns to specialization.

JEL Classification: C61, L16, L4, O31, O33.

\section{Introduction}

In this paper we analyze consumers' welfare as a function of a parameter representing the degree of competition. We consider the simplest endogenous growth R\&D based model reported in Barro and Sala-i-Martin (2004), Ch.6, which is a simplified version of Romer's (1990) model. In this setup $R \& D$ aims to expand the number of existing varieties of the intermediate goods and it uses labor. It is also assumed that intermediate goods are not durable.

By starting from the decentralized economy analysis we are able to derive the expression for consumers' welfare since we provide the closed form solutions for the trajectories of all the key variables. Usually economists have studied non-linear dynamical systems of endogenous growth models in a qualitative way, that is using the phase diagram and completed this

\footnotetext{
*Université catholique de Louvain, IRES, B-1348 Louvain-la-Neuve, Belgium and University of Rome "La Sapienza", Faculty of Economics, I-00161, Rome, Italy. Email: daria.onori@uclouvain.be.

I wish to thank R. Boucekkine, C. Camacho, A. Venditti, E. Marchetti, and the partecipants in seminars and conferences at Université catholique de Louvain and GREQAM Marseilles. Grateful acknowledgement is made for financial support by GREQAM. The usual disclaimer applies.
} 
analysis with a quantitative one, linearizing around the steady state after having applied the so called reduction dimension strategy. This technique consists in the definition of new variables in terms of ratio between the original ones and it is generally not sufficient to be fully conclusive about the dynamic properties of the original variables in levels. We solve instead the dynamical system analytically. In particular we derive the differential equation which describes the dynamics of the amount of labor used in $R \& D$. We obtain a Riccati differential equation which admits two steady states: one is the balanced growth path solution of Barro and Salai-Martin Ch. 6, and it is unstable, the other, which is equal to the total labor force, which is stable. After solving it we provide the explicit solution for the trajectory of consumption. We solve for the optimal trajectories by using the transversality condition and we find that for it to be satisfied the labor force allocated in R\&D at time zero must be set equal to its balanced growth path solution. This proves that the model does not exhibit transitional dynamics. We can also determine the initial value for consumption by using market clearing condition for the final good at time zero. This allows us to compute the value of consumers' welfare, a result that is not usual to be found in literature, given that models usually do not allow for closed form solutions. We then solve the social planner's maximization problem and derive also in this case the level of consumption and the related welfare function.

The previous results enable us to undertake a comparative static analysis. In this model an increase in market power, represented by a reduction of the parameter $\alpha$, reduces the decentralized economy's growth rate, since it causes a reallocation of the fixed labor force toward the final good sector, slowing down the R\&D production, which in turns constitutes the engine of growth. This result places this model in the debate on the relationship between competition and growth in R\&D-Based Growth Models. The first generation of Schumpeterian growth models of vertical differentiation (Grossman and Helpman 1991, Aghion and Howitt 1992, Barro and Sala-i-Martin 2004) predicts that innovation and hence growth should decline with competition, because more competition reduces the rents that reward successful innovators (this is the so called Schumpeterian effect). This discourages firms from investing in $\mathrm{R} \& \mathrm{D}$, thus reducing the innovation rate and as a consequence the long run growth rate of the economy. However the empirical literature of the Nineties (Gerosky 1995, Blundell, Griffith and Van Reenen 1999) suggests a positive correlation between competition and growth. On the contrary models of horizontal differentiation are less conclusive on the predictions. The model considered in this paper (Romer's 1990 model) predicts a positive relationship between competition and growth, while Grossman and Helpman's (1991, Chapter 3) model predicts a negative relationship (see Bucci 2003 for a review). Recently it has been shown some evidence in favor of a bell- shaped relationship between competition and growth (Aghion et al. 2005). Few are the explanations provided by the literature. For example Aghion et al. (2005) explain the bell-shaped form by means of the interaction between the Schumpeterian effect and the escape competition effect, while for Bucci (2005) the interaction is between a resource 
allocation effect and the Schumpeterian effect ${ }^{1}$. Finally, Onori's (2010) explanation relies on the presence of a spillover effect stemming from the intermediate good sector that may prevail over the Schumpeterian effect.

On the other hand, the social planner's growth rate is independent from market power index because she corrects this market failure. Hence we would expect a positive effect of an increase in $\alpha$ on consumers' welfare in the decentralized economy and no effect on social planner's welfare. However we surprisingly find that, when the total labor force is greater than a certain level, consumers' welfare in the decentralized economy is an inverted-N shaped function of $\alpha$, while the welfare level of the centralized economy is a monotonically decreasing function of $\alpha$. This suggests that there exists an additional effect which interacts with market power decrease. This effect is negative and is represented by the decrease of the degree of returns to specialization. The degree of returns to specialization measures the degree to which the society benefits from "specializing" production between a larger number of intermediates. Hence in the social planner's case an increase in $\alpha$ simply reduces the degree of returns to specialization, so explaining the monotonically decreasing welfare function. In the decentralized economy the negative effect of a reduction of the degree of returns to specialization may prevail over the positive effect of a reduction of market power, giving raise to an inverted-N shaped function.

The structure of the paper is the following. In section 2 we briefly present the model and we derive the closed form solutions for labor force employed in $R \& D$ and for consumption in the decentralized economy and in the social planner's case. Section 3 provides the welfare analysis, finally section 4 concludes.

\section{The model}

\subsection{The decentralized economy}

In this section we briefly describe the model of horizontal differentiation shown in Ch. 6 of Barro and Sala-i-Martin (2004), in the decentralized economy case.

There are three different productive sectors. The undifferentiated final good is produced by using labor and intermediated goods and it is sold in a perfectly competitive market ${ }^{2}$. The technology is represented by a Cobb-Douglas production function:

$$
Y(t)=\left(L-L_{R}(t)\right)^{1-\alpha} \sum_{j=1}^{N(t)}\left(x_{j}(t)\right)^{\alpha}
$$

\footnotetext{
${ }^{1}$ Bucci (2005) generalizes the model considered in the present paper to the case in which labor is used also in the production of the intermediate goods.

${ }^{2}$ We take it as the numeraire.
} 
where $\left(L-L_{R}(t)\right)$ is the work force used in the final good production ${ }^{3}, x_{j}(t)$ is the quantity employed of the $j t h$ intermediate good and $0<\alpha<1$. The total labor force $L$ is constant over time.

The first order conditions of final good producers' determine the demand function for each intermediate good and the wage rate:

$$
\begin{gathered}
x_{j}(t)=\left(\frac{\alpha}{p_{j}}\right)^{\frac{1}{1-\alpha}}\left(L-L_{R}(t)\right) \\
w(t)=(1-\alpha) Y(t) /\left(L-L_{R}(t)\right) .
\end{gathered}
$$

R\&D firms face a two-stage decision process. In the first stage, they decide whether to devote resources to invent a new brand. They will invest until the point in which the expected flow of future profits equals the R\&D cost. In the second stage, the inventors, provided by a perpetual patent, enter the intermediate good sector and chose the optimal price at which to sell their new product. Hence the intermediate goods industry is imperfectly competitive and each producer uses the same technology: one unit of the final good is needed to produce one unit of intermediate good. Hence the profit of each monopolist is given by $\pi_{j}(t)=p_{j} x_{j}(t)-x_{j}(t)$ and the monopolistic price is $p_{j}=\frac{1}{\alpha}, \forall j$. Using these informations we obtain the produced quantity of each intermediate good $x_{j}(t)=x(t)=\left(L-L_{R}(t)\right) \alpha^{\frac{2}{1-\alpha}}, \forall j$.

The $R \& D$ sector is perfectly competitive and there is a positive spillover effect attached to the available stock of disembodied knowledge (approximated by the existing number of designs, $N(t)$ ) in discovering a new product variety: that is to produce a new variety of good it is necessary to employ $\frac{\eta}{N(t)}$ units of labor and $\eta>0$ represents a cost parameter. Hence the dynamics of the number of brands is

$$
\dot{N}(t)=\frac{L_{R}(t)}{\eta} N(t) .
$$

As the research sector is perfectly competitive, new firms will enter each $R \& D$ race until the profit is zero. As Barro and Sala-i-Martin analysis is only focused on the balanced growth path, $L_{R}$ and the interest rate are constant, so the research arbitrage condition writes as

$$
\int_{t}^{+\infty} \frac{1-\alpha}{\alpha}\left(L-L_{R}\right) \alpha^{\frac{2}{1-\alpha}} e^{-r(\nu-t)} d \nu=\eta(1-\alpha) \alpha^{\frac{2 \alpha}{1-\alpha}}
$$

The left hand side represents the benefit of each innovator while the right and side is the cost of doing $\mathrm{R} \& \mathrm{D}^{4}$. It can be rewritten as

\footnotetext{
${ }^{3}$ It can be interpreted as human capital.

${ }^{4}$ Since in this model labor is homogeneous and perfectly mobile, the wage rate is the same in the final good sector and in the $\mathrm{R} \& \mathrm{D}$ sector.
} 


$$
r=\frac{\alpha\left(L-L_{R}\right)}{\eta}
$$

Finally the representative household maximizes utility over an infinite horizon. It consumes the final good and her savings finance R\&D

$$
\begin{array}{cc}
\max _{C(t)} \quad U=\int_{0}^{+\infty} \frac{C(t)^{1-\sigma}}{1-\sigma} e^{-\rho t} d t \\
\text { s. t. } \quad \dot{a}(t)=r(t) a(t)+w(t) L-C(t)
\end{array}
$$

The optimality conditions are thus given by

$$
\left\{\begin{array}{ccc}
\frac{\dot{C}(t)}{C(t)} & = & \frac{1}{\sigma}(r(t)-\rho) \\
\dot{a}(t) & = & r(t) a(t)+w(t) L-C(t) \\
\lim _{t \rightarrow+\infty} C(t)^{-\sigma} a(t) e^{-\rho t} & = & 0
\end{array}\right.
$$

In a general equilibrium setup the following relation between the consumer's assets and the number of varieties exists: $a(t)=N(t) \eta(1-\alpha) \alpha^{\frac{2 \alpha}{1-\alpha}}$.

Given the market clearing condition for consumption good and the fact that the aggregate output is proportional to the number of varieties $\left(Y(t)=N(t)\left(L-L_{R}(t)\right) \alpha^{\frac{2 \alpha}{1-\alpha}}\right)$, in the balanced growth path (BGP hereafter) all the variables grow at the same positive and constant growth rate:

$$
g_{Y}=g_{C}=g_{X}=g_{N}=g=\frac{\bar{L}_{R}}{\eta}
$$

where $\bar{L}_{R}$ is the steady state solution for the labor employed in R\&D and it is given by

$$
\bar{L}_{R}=\frac{\eta}{\alpha+\sigma}\left(\frac{\alpha}{\eta} L-\rho\right)
$$

\subsection{The closed form solutions}

In this section we derive the optimal trajectories for the labor force employed in R\&D and consumption. We prove that the model does not exhibit transitional dynamics.

In order to derive the dynamics of $L_{R}(t)$ we differentiate the research arbitrage condition

$$
\int_{t}^{+\infty} \frac{1-\alpha}{\alpha}\left(L-L_{R}(\nu)\right) \alpha^{\frac{2}{1-\alpha}} e^{-\bar{r}(t, \nu)(\nu-t)} d \nu=\eta(1-\alpha) \alpha^{\frac{2 \alpha}{1-\alpha}}
$$

with respect to time. We remind that in this case the interest rate is the average over the period: $\bar{r}(t, \nu)=\frac{1}{\nu-t} \int_{t}^{\nu} r(\omega) d \omega$ and the amount of human capital employed in R\&D can vary over time. 
Let us call the R\&D benefit $B(t)$ :

$$
B(t) \equiv \int_{t}^{+\infty} \frac{1-\alpha}{\alpha}\left(L-L_{R}(\nu)\right) \alpha^{\frac{2}{1-\alpha}} e^{-\bar{r}(t, \nu)(\nu-t)} d \nu
$$

So

$$
\frac{d B(t)}{d t}=-\frac{1-\alpha}{\alpha}\left(L-L_{R}(t)\right) \alpha^{\frac{2}{1-\alpha}}+r(t) B(t)
$$

We know by the research arbitrage condition that $B(t)=\eta(1-\alpha) \alpha^{\frac{2 \alpha}{1-\alpha}}$, hence $(2.1)$ becomes

$$
r(t)=\frac{\alpha\left(L-L_{R}(t)\right)}{\eta}
$$

We can rewrite the Euler equation as

$$
\frac{\dot{C}(t)}{C(t)}=\frac{r(t)-\rho}{\sigma}=\frac{\frac{\alpha\left(L-L_{R}(t)\right)}{\eta}-\rho}{\sigma} .
$$

In order to obtain the differential equation describing the dynamics $L_{R}(t)$, we use the final good market clearing condition:

$$
Y(t)=C(t)+N(t) x(t),
$$

where $X(t)=N x(t)$ is the aggregate quantity of produced intermediate goods.

Since the quantity produced by each intermediate firm is given by $x(t)=\left(L-L_{R}(t)\right) \alpha^{\frac{2}{1-\alpha}}$, we can rewrite the final output as $Y(t)=N(t)\left(L-L_{R}(t)\right) \alpha^{\frac{2 \alpha}{1-\alpha}}$. Using these informations, we can solve the final good market clearing condition with respect to consumption

$$
C(t)=\alpha^{\frac{2 \alpha}{1-\alpha}}\left(1-\alpha^{2}\right) N\left(L-L_{R}(t)\right)
$$

By differentiating it with respect to time we obtain

$$
\dot{C}(t)=\alpha^{\frac{2 \alpha}{1-\alpha}}\left(1-\alpha^{2}\right)\left[\dot{N}(t)\left(L-L_{R}(t)\right)-\dot{L}_{R}(t) N(t)\right] .
$$

Hence

$$
\frac{\dot{C}(t)}{C(t)}=\frac{\dot{N}(t)}{N(t)}-\frac{\dot{L}_{R}(t)}{L-L_{R}(t)} .
$$

Since the growth rate of designs is $\frac{\dot{N}(t)}{N(t)}=\frac{L_{R}(t)}{\eta}$, the previous relation becomes

$$
\frac{\dot{C}(t)}{C(t)}=\frac{L_{R}(t)}{\eta}-\frac{\dot{L}_{R}(t)}{L-L_{R}(t)} .
$$


By equating (2.3) and (2.4) we get a first order differential equation in $L_{R}$ :

$$
\dot{L}_{R}(t)=\frac{1}{\eta}\left[\left(-1-\frac{\alpha}{\sigma}\right) L_{R}^{2}(t)+\left(L+\frac{2 \alpha L}{\sigma}-\frac{\rho \eta}{\sigma}\right) L_{R}(t)+\frac{\rho \eta L}{\sigma}-\frac{\alpha L^{2}}{\sigma}\right] .
$$

This equation admits two steady states, which are given by

$$
\bar{L}_{R_{1}}=\frac{\eta}{\alpha+\sigma}\left(\frac{\alpha}{\eta} L-\rho\right)
$$

which is the solution we get with the BGP analysis, and

$$
\bar{L}_{R_{2}}=L
$$

so that in this case the economy would end up starving in the long run.

\subsubsection{The trajectories of $L_{R}(t)$ and $C(t)$}

In this section we derive the trajectories of $L_{R}(t)$ and $C(t)$. Equation (2.5) is a Riccati differential equation. In order to solve it we need to guess a particular solution. The particular solutions are the ones that solves the second degree polynomial on the right hand side, that is the steady state solutions.

We start by guessing $\tilde{L}_{R}=\bar{L}_{R_{2}}=L$, hence the general solution is given by

$$
L_{R}(t)=L+\left[d e^{\frac{1}{\eta}\left(L+\frac{\rho \eta}{\sigma}\right) t}-\frac{1+\frac{\alpha}{\sigma}}{L+\frac{\rho \eta}{\sigma}}\right]^{-1}
$$

The integration constant $d$ is given by

$$
d=\left[L_{R}(0)-L\right]^{-1}+\frac{1+\frac{\alpha}{\sigma}}{L+\frac{\rho \eta}{\sigma}}
$$

where $L_{R}(0)$, the initial value for the work force used in $R \& D$, is not known in advance since $L_{R}(t)$ is not a predetermined variable ${ }^{5}$. Equation (2.6) becomes

$$
L_{R}(t)=L+\left[\left[\left(L_{R}(0)-L\right)^{-1}+\frac{1+\frac{\alpha}{\sigma}}{L+\frac{\rho \eta}{\sigma}}\right] e^{\frac{1}{\eta}\left(L+\frac{\rho \eta}{\sigma}\right) t}-\frac{1+\frac{\alpha}{\sigma}}{L+\frac{\rho \eta}{\sigma}}\right]^{-1} .
$$

If we take the limit for $t \rightarrow+\infty$ we obtain

$$
\lim _{t \rightarrow+\infty} L_{R}(t)=L=\bar{L}_{R_{2}}
$$

\footnotetext{
${ }^{5}$ Notice that this solution is possible if and only if $L_{R}(0) \neq L$.
} 


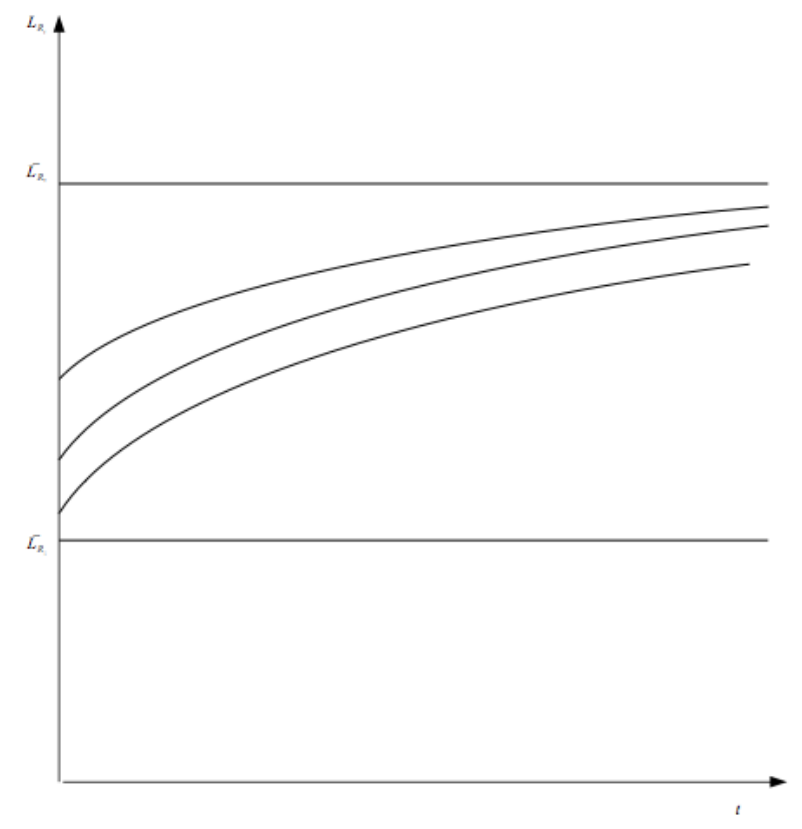

Figure 2.1: Possible trajectories of $L_{R}(t)$

that is the steady state $\bar{L}_{R_{2}}=L$ is stable while the BGP solution, $\bar{L}_{R_{1}}=\frac{\eta}{\alpha+\sigma}\left(\frac{\alpha}{\eta} L-\rho\right)$, is unstable $^{6}$.

Graphically the possible trajectories, for each possible initial value $L_{R}(0)$, of $L_{R}(t)$ are given by

Our aim is to determine the optimal trajectory among these.

For sake of simplicity the analysis will be conducted for the logarithmic utility case $(\sigma=1)$.

By using (2.4), (2.5), (2.7) we can find the expression for the trajectory of consumption:

$$
\begin{aligned}
C(t) & =f \exp \left[\frac{L-\rho \eta}{2 \eta(1+\alpha)} t\right]\left\{\frac{1+\alpha}{L+\rho \eta}-\left[\left(L_{R}(0)-L\right)^{-1}+\frac{1+\alpha}{L+\rho \eta}\right] \exp \left[\frac{1}{\eta}(L+\rho \eta) t\right]\right\}^{-\frac{\alpha}{1+\alpha}} \\
& =f \exp \left[\frac{\bar{L}_{R_{1}}}{\eta} t\right]\left\{\frac{1+\alpha}{L+\rho \eta}-\left[\left(L_{R}(0)-L\right)^{-1}+\frac{1+\alpha}{L+\rho \eta}\right] \exp \left[\frac{1}{\eta}(L+\rho \eta) t\right]\right\}^{-\frac{\alpha}{1+\alpha}}
\end{aligned}
$$

The integration constant $f$ is given by

$$
f=C(0)\left(L-L_{R}(0)\right)^{\frac{\alpha}{1+\alpha}}
$$

where consumption at time zero, $C(0)$, has to be determined using the optimality conditions.

\footnotetext{
${ }^{6}$ These results are confirmed by the linearization around each steady state.
} 


\subsubsection{Optimal trajectories}

In the following proposition we prove the optimality of the trajectory $L_{R}(t)=\bar{L}_{R_{1}}=$ $\frac{\alpha L-\eta \rho}{1+\alpha}, \forall t$, that is the economy immediately jumps on the BGP.

Proposition 1. The trajectory $L_{R}(t)=\bar{L}_{R_{1}}=\frac{\alpha L-\eta \rho}{1+\alpha}, \forall t$, obtained for $L_{R}(0)=\bar{L}_{R_{1}}=\frac{\alpha L-\eta \rho}{1+\alpha}$ is optimal.

Proof. In order to be optimal the solutions for $L_{R}(t)$ and $C(t)$ given by, respectively, (2.7) and (2.8) have to satisfy the transversality condition. It is given by

$$
\lim _{t \rightarrow+\infty} e^{-\rho t} \frac{1}{C(t)} a(t)=0
$$

The total of consumer's assets equal the market value of the intermediate firms:

$$
a(t)=V N(t)=\eta(1-\alpha) \alpha^{\frac{2 \alpha}{1-\alpha}} N(t)
$$

so the transversality condition can be rewritten as

$$
\lim _{t \rightarrow+\infty} \eta(1-\alpha) \alpha^{\frac{2 \alpha}{1-\alpha}} e^{-\rho t} \frac{1}{C(t)} N(t)=0
$$

Plugging in equations (2.7) and (2.8) we obtain:

$$
\begin{gathered}
\lim _{t \rightarrow+\infty}\left\{N(t) \eta(1-\alpha) \alpha^{\frac{2 \alpha}{1-\alpha}} \exp [-\rho t] \frac{1}{f}\left[\exp \left[\frac{\bar{L}_{R_{1}}}{\eta} t\right]\right]^{-1}\left\{\frac{1+\alpha}{L+\rho \eta}-d \exp \left[\frac{1}{\eta}(L+\rho \eta) t\right]\right\}^{\frac{\alpha}{1+\alpha}}\right. \\
\left.\exp \left[\frac{L}{\eta}\right] \exp \left[\frac{\left[d \exp \left[\frac{1}{\eta}(L+\rho \eta) t\right]-\frac{1+\alpha}{L+\rho \eta}\right]^{-1}}{\eta}\right]\right\}=0
\end{gathered}
$$

where $d=\left[L_{R}(0)-L\right]^{-1}+\frac{1+\alpha}{L+\rho \eta}$ and $f=C(0)\left(L-L_{R}(0)\right)^{\frac{1-\alpha}{1+\alpha}}$. Then

$$
\lim _{t \rightarrow+\infty}\left\{\frac{1+\alpha}{L+\rho \eta}-d \exp \left[\frac{1}{\eta}(L+\rho \eta) t\right]\right\}^{\frac{\alpha}{1+\alpha}}= \pm \infty
$$

depending on the sign of the constant $d^{7}$.

Since all the remaining terms tend to a constant or to zero, for the transversality condition to be satisfied a necessary condition is to have $d=0 \Longleftrightarrow L_{R}(0)=\bar{L}_{R_{1}}$. Given this, we can

\footnotetext{
${ }^{7}$ The constant $d$ is negative for $\bar{L}_{R_{1}}<L_{R}(0)<\bar{L}_{R_{2}}$, it is positive for $0<L_{R}(0)<\bar{L}_{R_{1}}$, it is equal to zero for $L_{R}(0)=\bar{L}_{R_{1}}$, it is infinite for $L_{R}(0)=\bar{L}_{R_{2}}$.
} 
determine the other constant $f$ using the final good market clearing condition at $t=0$ :

$$
\begin{gathered}
Y(0)=C(0)+N(0) X(0) \\
C(0)=\alpha^{\frac{2 \alpha}{1-\alpha}}\left(1-\alpha^{2}\right) N(0)\left(L-L_{R}(0)\right) \\
C(0)=\alpha^{\frac{2 \alpha}{1-\alpha}}\left(1-\alpha^{2}\right) N(0)\left(L-\bar{L}_{R_{1}}\right)
\end{gathered}
$$

where $C(0)$ is given by equation $(2.9): C(0)=f\left(L-L_{R}(0)\right)^{\frac{\alpha}{1+\alpha}}=f\left(L-\bar{L}_{R_{1}}\right)^{\frac{\alpha}{1+\alpha}}$

Hence

$$
\begin{gathered}
f=\alpha^{\frac{2 \alpha}{1-\alpha}} f\left(L-\bar{L}_{R_{1}}\right)^{-\frac{\alpha}{1+\alpha}}\left(1-\alpha^{2}\right) N(0)\left(L-\bar{L}_{R_{1}}\right) \\
=\alpha^{\frac{2 \alpha}{1-\alpha}}\left(1-\alpha^{2}\right) N(0)\left(L-\bar{L}_{R_{1}}\right)^{\frac{1}{1+\alpha}} \\
=\alpha^{\frac{2 \alpha}{1-\alpha}}\left(1-\alpha^{2}\right) N(0)\left(\frac{L+\rho \eta}{1+\alpha}\right)^{\frac{1}{1+\alpha}}
\end{gathered}
$$

In Proposition 1 we have just found a necessary condition for the transversality condition to be satisfied. Actually there can be other values for the constant $C(0)$ which satisfy it. To see this, we first compute $\exp [-\rho t] \frac{1}{f}\left[\exp \left[\frac{\bar{L}_{R_{1}}}{\eta} t\right]\right]^{-1}=\exp \left[\frac{-\rho-\alpha \rho-\alpha L+\rho \eta}{1+\alpha} t\right]$. Then combining it with $\left\{\frac{1+\alpha}{L+\rho \eta}-d \exp \left[\frac{1}{\eta}(L+\rho \eta) t\right]\right\}^{\frac{\alpha}{1+\alpha}}$, we require that

$$
\exp \left[\left[\frac{-\rho-\alpha \rho-\alpha L+\rho \eta}{1+\alpha} t\right]^{\frac{1+\alpha}{\alpha}}+\left[\frac{1}{\eta}(L+\rho \eta) \mathrm{t}\right]\right] \rightarrow 0 \text {. }
$$

In light of Proposition 1, the trajectory of consumption can be rewritten as

$$
C(t)=\alpha^{\frac{2 \alpha}{1-\alpha}}(1-\alpha) N(0)(L+\rho \eta) \exp \left[\frac{\alpha L-\rho \eta}{\eta(1+\alpha)} t\right] .
$$

Hence the initial level of consumption is optimally chosen in such a way that the economy jumps on the BGP at $t=0$.

\subsection{The centralized economy}

In this section we derive the closed form solution for consumption of a benevolent social planner who seeks to maximize the representative consumer's utility. The social planner is constrained 
by the low of motion of designs and by the economy's budged constraint:

$$
\begin{array}{cc}
\max _{\left\{C(t), x(t), L_{R}(t)\right\}} & \int_{0}^{+\infty} \ln C(t) e^{-\rho t} d t \\
\text { s. t. } & Y(t)=C(t)+N(t) x(t) \\
\dot{N}(t) & =\frac{L_{R}(t)}{\eta} N(t) \\
& N(0) \text { given }
\end{array}
$$

From the economy's resource constraint we can derive an expression for consumption level

$$
C(t)=\left(L-L_{R}(t)\right)^{1-\alpha} N(t) x(t)^{\alpha}-N(t) x(t)
$$

so that the problem can be rewritten as

$$
\begin{gathered}
\left.\max _{\left\{x(t), L_{R}(t)\right\} \quad \int_{0}^{+\infty} \ln \left\{\left(L-L_{R}(t)\right)^{1-\alpha}\right.} N(t) x(t)^{\alpha}-N(t) x(t)\right\} e^{-\rho t} d t \\
\dot{N}(t)=\frac{L_{R}(t)}{\eta} N(t) \\
N(0) \text { given }
\end{gathered}
$$

The Pareto optimal solution for $x(t), L_{R}(t)$ and the growth rate are given by

$$
\begin{gathered}
x_{S O}=\left(L-L_{R}\right) \alpha^{\frac{1}{1-\alpha}} \\
L_{R_{S O}}=L-\rho \eta \\
g_{S O}=\frac{L}{\eta}-\rho .
\end{gathered}
$$

Hence equation (2.12) can be rewritten as

$$
C(t)_{S O}=\frac{1-\alpha}{\alpha} \alpha^{\frac{1}{1-\alpha}} \rho \eta N(0) \exp \left[\left(\frac{L}{\eta}-\rho\right) t\right] .
$$

\section{Welfare, competition, specialization and growth}

In this section we analyze the effects of a change in $\alpha$ on consumers' welfare of the decentralized economy and compare them with the results of the centralized economy.

The parameter $\alpha$ plays two different roles in this model: first $\frac{1}{\alpha}-1$ is the mark up enjoyed by innovators, so an increase in $\alpha$ reduces their market power; second it determines the degree of returns to specialization ${ }^{8}$.

\footnotetext{
${ }^{8}$ In this framework $\alpha$ cannot represent the consumer's love for variety since by the aggregate resource
} 
Definition. (Degree of returns to specialization (Benassy 1998)). The degree of returns to specialization measures the degree to which the society benefits from "specializing" production between a larger number of intermediates $N(t)$.

We now compute it for our economy. Since the intermediate industries are symmetric, the production function of the final good can be written as

$$
Y(t)=\left(L-L_{R}(t)\right)^{1-\alpha} \sum_{j=1}^{N(t)}\left(x_{j}(t)\right)^{\alpha}=\left(L-L_{R}(t)\right)^{1-\alpha} N(t) x(t)^{\alpha}
$$

Given that the aggregate quantity of intermediate goods is $X(t)=N(t) x(t)$ the previous expression rewrites as

$$
Y(t)=\left(L-L_{R}(t)\right)^{1-\alpha} N(t)^{1-\alpha} X(t)^{\alpha}
$$

where $(1-\alpha)$ is the degree of returns to specialization. Hence an increase in $\alpha$ has a negative effect on it $^{9}$.

\subsection{The growth rates}

For sake of comparison we report the expression of the growth rates of the decentralized economy and of the centralized economy:

$$
g_{D E}=\frac{\alpha L-\rho \eta}{\eta(1+\alpha)} \text { and } g_{S O}=\frac{L}{\eta}-\rho
$$

We immediately note that $\frac{\partial g_{D E}}{\partial \alpha}>0$ while the social planner's growth rate is independent from $\alpha$. This means that the growth rate of the decentralized economy only depends on market power, given that the social planner eliminates this distortion. An increase in $\alpha$ rises the growth rate of the decentralized economy because a reduction of the mark ups of all the intermediate inputs makes it more profitable for the final good producer to substitute labor force with intermediate goods. As a consequence, given that the total labor force is constant, the quantity of human capital available for research increases and since this sector is the engine of growth, the growth rate rises.

On the contrary, we will see in the next section that the degree of returns to specialization plays a role on determining welfare.

constraint consumption is given by $C(t)=\left(L-L_{R}(t)\right)^{1-\alpha} \sum_{j=1}^{N(t)}\left(x_{j}(t)\right)^{\alpha}-N(t) x(t)$, so it depends also linearly on $x(t)$.

${ }^{9}$ We remark that for a given $N(t)$ the production function exhibits constant returns to scale in $L(t)$ and $X(t)$. 


\subsection{The Welfare functions}

In this section we prove that the relationship between welfare and $\alpha$ is monotonically decreasing in the centralized economy, while it can be monotonically decreasing or an inverted-N shape in the decentralized economy and we provide an economic interpretation.

Proposition 2. The relationship between consumers' welfare and $\alpha$ in the centralized economy is monotonically decreasing.

Proof. The optimal level of consumption for the centralized economy is given by

$$
C(t)_{S O}=\frac{1-\alpha}{\alpha} \alpha^{\frac{1}{1-\alpha}} \rho \eta N(0) \exp \left[\left(\frac{L}{\eta}-\rho\right) t\right]
$$

hence welfare is

$$
U_{S O}^{*}=\frac{1}{\rho}\left\{\ln \left[\frac{1-\alpha}{\alpha} \alpha^{\frac{1}{1-\alpha}} \rho \eta N(0)\right]+\frac{1}{\rho}\left(\frac{L}{\eta}-\rho\right)\right\} .
$$

If we derive it with respect to $\alpha$ we obtain

$$
\frac{\partial U_{S O}^{*}}{\partial \alpha}=\frac{1}{\rho} \frac{1}{1-\alpha}\left[\frac{1}{1-\alpha} \ln \alpha\right]
$$

which is always negative since $\alpha \in(0,1)$.

The results for the decentralized economy are reported in the following Proposition:

Proposition 3. If $L \geq \underline{L}$, where $\underline{L}=\lambda \rho \eta, \lambda \equiv f\left(\min \left(-\frac{1}{1-\alpha}\left[\frac{2}{1-\alpha} \ln \alpha+1\right]\right)\right){ }^{10}$, the relationship between $\alpha$ and the consumer's welfare of the decentralized economy is an inverted- $N$ shape, otherwise it is a decreasing function of $\alpha$.

Proof. (Cf. Appendix).

In Section 3.1 we saw that the growth rate of the decentralized economy is an increasing function of $\alpha$ while the one of the centralized economy is independent from $\alpha$. This result is linked to social planner's effort to correct the distortion induced by monopoly. The results showed in Proposition 2 and Proposition 3 suggest that there are two interplaying effect on welfare stemming from an increase in $\alpha$ :

- a positive effect: an increase in $\alpha$ reduces innovators' market power ("competition effect");

- a negative effect: an increase in $\alpha$ reduces the degree of returns to specialization ("degree of returns to specialization effect").

\footnotetext{
${ }^{10} \lambda \simeq 5.54394$
} 
The relationship between welfare and $\alpha$ in the social planner case is negative and this means that only the negative effect of a reduction of the degree of returns to specialization plays a role, since the social planner corrects the market imperfection. While the relationship between welfare and $\alpha$ may be an inverted-N shape in the case of the decentralized economy. We can disentangle these two effects by looking at the derivative of the welfare function of the decentralized economy

$$
\frac{\partial U_{D E}^{*}}{\partial \alpha}=\frac{1}{\rho}\left\{\frac{1}{1-\alpha}\left[\frac{2}{1-\alpha} \ln \alpha+1\right]+\frac{1}{\rho \eta} \frac{L+\rho \eta}{(1+\alpha)^{2}}\right\}
$$

It can be decomposed in two parts:

$$
g(\alpha) \equiv \frac{1}{1-\alpha}\left[\frac{2}{1-\alpha} \ln \alpha+1\right]
$$

and

$$
h(\alpha) \equiv \frac{1}{\rho \eta} \frac{L+\rho \eta}{(1+\alpha)^{2}} .
$$

These two functions are represented in the figure below
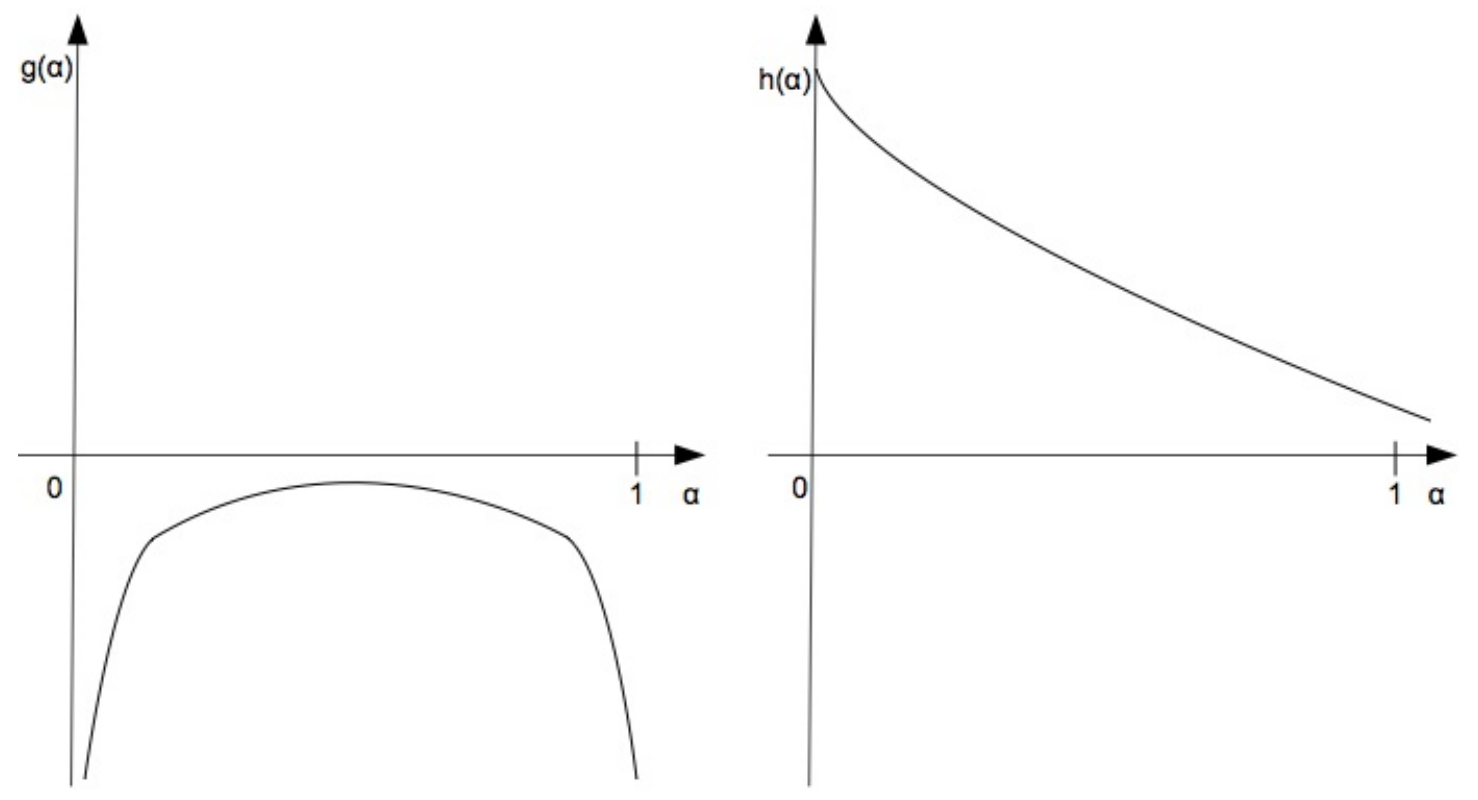

Figure 3.1: Degree of returns to specialization effect $(g(\alpha))$ and Competition effect $(h(\alpha))$

Since $g(\alpha)$ is negative, it clearly represents the degree of returns to specialization effect, 
while being $h(\alpha)$ positive, it can be identified as the competition effect. The presence of these two effects in the welfare function of the decentralized economy becomes much more evident if we compare equation (3.2) with the derivative of the welfare function in the social planner's case, as given by equation (3.1). The latter function shows the same behavior as $g(\alpha)$, while we have already underlined that the competition effect is not present in the centralized economy, hence we do not observe the part which depends positively on $\alpha$.

The position of $h(\alpha)$ in the graph depends on the values of the parameters $L, \eta$ and $\rho$ : as it is showed by the sufficient condition provided by Proposition 3, the greater is $L$ and the lower are $\eta$ and $\rho$, the greater is the possibility that $h(\alpha)$ and the symmetric of $g(\alpha)$ cross twice $^{11}$, hence the first derivative has two zeros and the welfare function is an inverted-N shape.

Graphically:

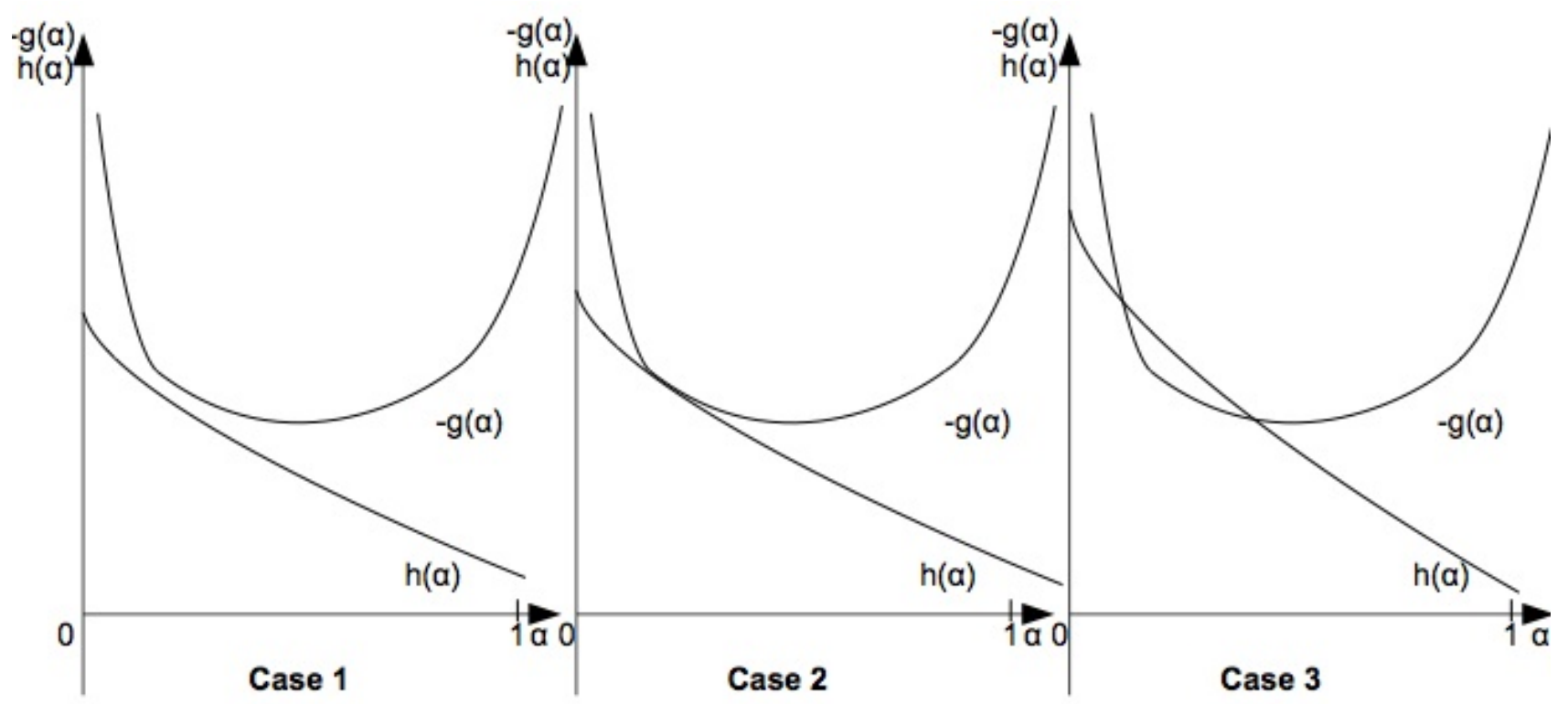

Figure 3.2: Interaction between Degree of returns to specialization effect and Competition effect

$L$ is the economy's total human capital. A high $L$ means that there will be more labor force to be employed in $R \& D^{12}$. But this is exactly the scale effect, a well known shortcoming of this model. $\eta$ is the R\&D parameter cost. The smaller is $\eta$, the lower is the cost of R\&D and the higher will be the resources engaged in expanding brands. Finally, $\rho$ is the consumers' discount rate. The lower is $\rho$, the higher is consumers' willingness to save, and the higher is their investment in $\mathrm{R} \& \mathrm{D}^{13}$. These effects are assimilable to the effect of an increase of $\alpha$ on

\footnotetext{
${ }^{11}$ This pushes $h(\alpha)$ up in the graphic.

${ }^{12}$ Recall that it is: $\frac{\partial \bar{L}_{R_{1}}}{\partial L}>0$.

${ }^{13}$ We remind that in this economy consumers finance $R \& D$.
} 
the growth rate of the decentralized economy through a reduction of the mark up and explain the positive sloped branch of the welfare function.

We can also say more about how the two effects determine the shape of the welfare function in the decentralized economy. This explanation is related to the second derivative of the welfare function. The competition effect has a positive effect on welfare but it is decreasing in $\alpha$ : as competition increases, even if human capital resources to be employed in $\mathrm{R} \& \mathrm{D}$ are a lot, innovators loose any incentive to invest in R\&D because they are deprived of their market power. On the other hand the degree of returns to specialization is not monotonic in $\alpha$. Remember Figure 3.1. When $\alpha$ is small, the degree of returns to specialization is high, however the weight of the intermediate goods on the final good production function is low: the importance of the technological sector in the economy is small and the produced quantity of each intermediate good $x=\left(L-L_{R}(t)\right) \alpha^{\frac{2}{1-\alpha}}$ is very small. For this reason the degree of returns to specialization effect is strongly negative. As $\alpha$ increases, even if the degree of returns to specialization is still negative, the function $g(\alpha)$ attains a maximum: the degree of returns to specialization is still high (the maximum is attained for $\alpha_{M A X} \simeq 0.383012$ ), but now the importance of the technological sector is higher than before and this explains why the degree of returns to specialization effect becomes less negative. When instead $\alpha$ is high enough, the degree of returns to specialization becomes smaller and smaller, while the impact of the intermediate sector is very high. This explains the decreasing branch of the function $g(\alpha)$. To sum up, in the first decreasing branch of the welfare function the degree of returns to specialization effect prevails over the competition effect because it is true that the degree of returns to specialization is high, but the importance of the intermediate sector is small. In the increasing branch of the welfare function it is possible to see the positive effect of an increase in competition counterbalancing the negative effect of the degree of returns to specialization effect. The last branch is negatively sloped because the the economy is close to a situation of perfect competition, the importance of the technological sector has become very high so the degree of returns to specialization dominates the competition effect, hence we see the same behavior of the welfare function as in the centralized economy case.

\subsubsection{Numerical examples}

In this section we provide some numerical examples in order to show the possible shapes that the welfare in the decentralized economy can exhibit. We normalize the total human capital $L$ to one and we set the discount rate $\rho$ equal to $0.03^{14}$. Moreover we normalize the initial number of designs to one and we let the $R \& D$ cost parameter free to vary because it is not possible to calibrate it by using the balanced growth path restrictions since all of them involve $\alpha$ and we cannot fix it. By the sufficient condition of Proposition 3 that assures an inverted-N

\footnotetext{
${ }^{14}$ These are quite standard parametrizations.
} 
shaped function we have to require that $\eta \leq \frac{L}{\underline{L} \rho} \simeq 6.01257$. Figure 3.3 represents the derivative of the welfare function with respect to $\alpha$ and shows some values for $\eta$ to have an inverted-N function:

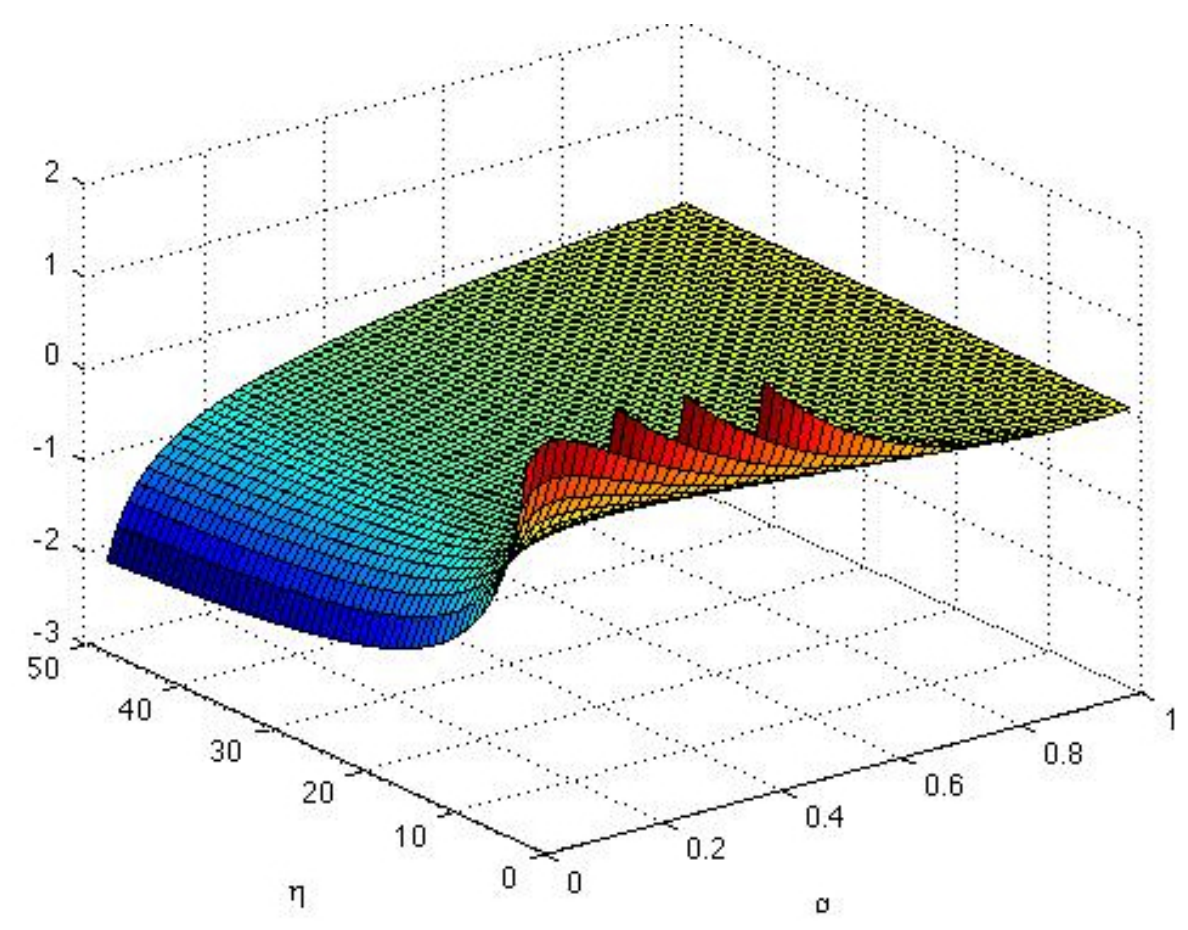

Figure 3.3: The sign of the first derivative $(L=1, \rho=0.03, N(0)=1)$

The following two figures show some examples in which the welfare function is decreasing and in which it is an inverted-N shape:

$$
\eta=10 \text { : }
$$




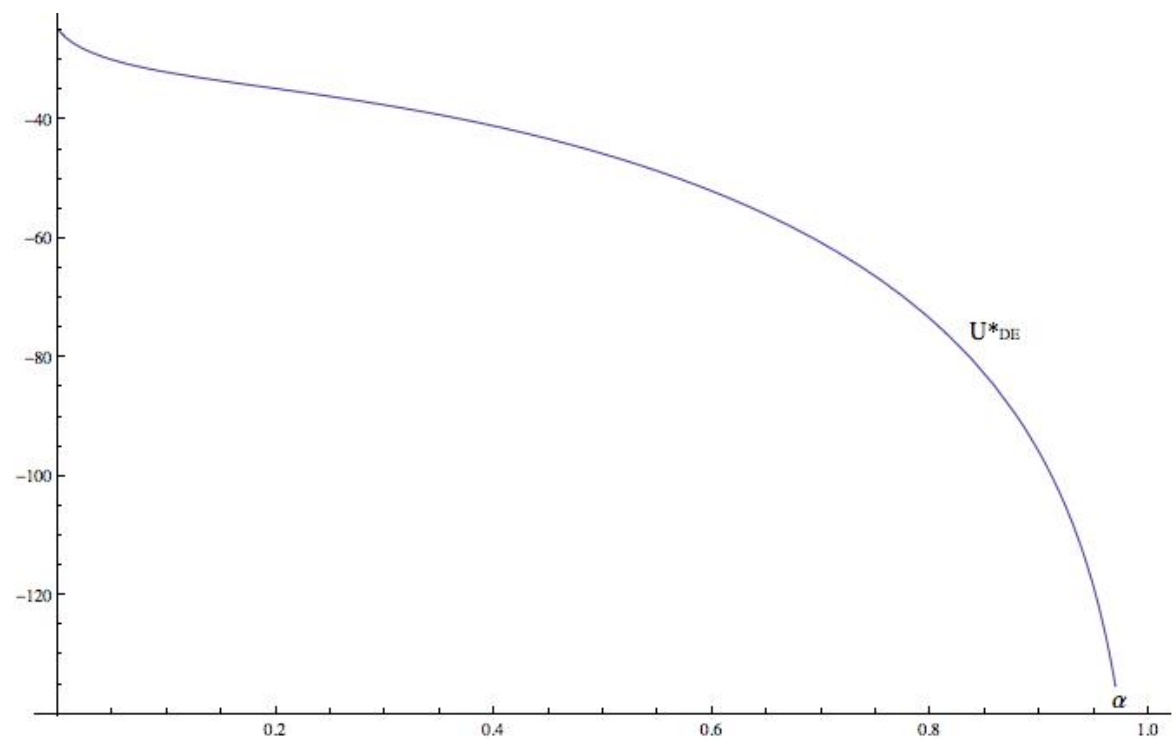

Figure 3.4: $\eta=10$ : The welfare function is monotonically decreasing

$\eta=5:$

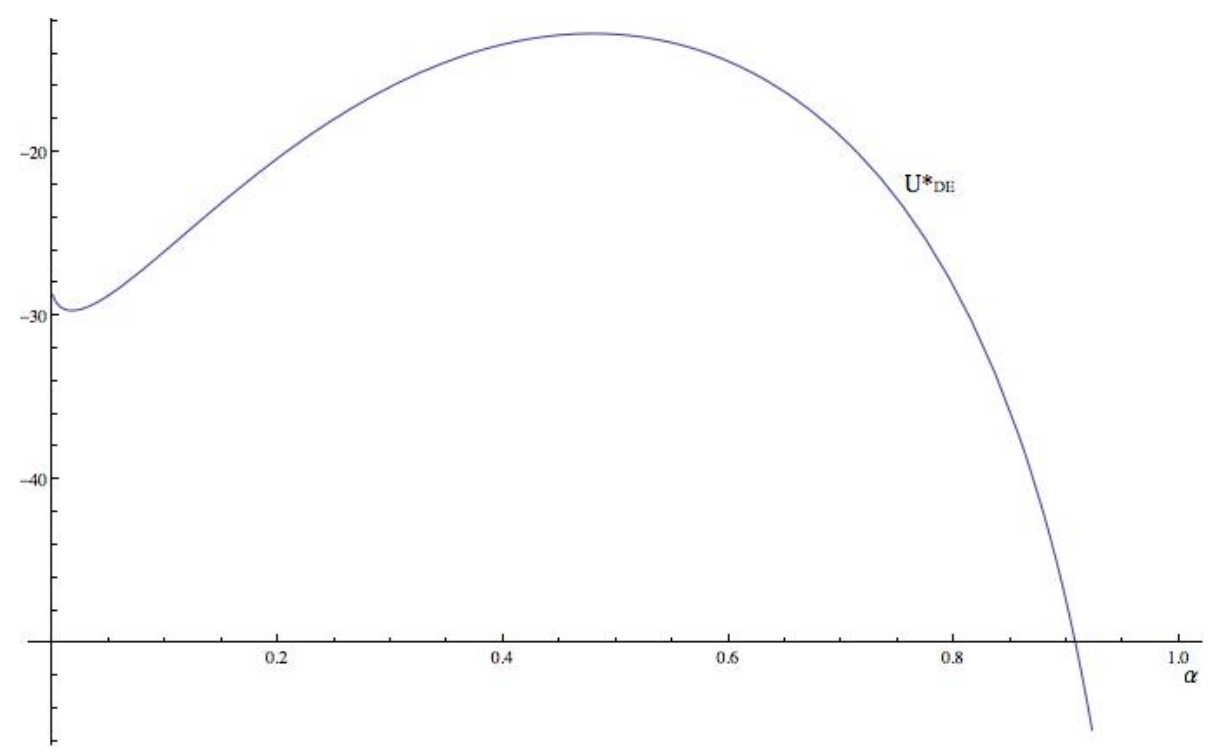

Figure 3.5: $\eta=5$ : The welfare function is an inverted-N shape

$$
\eta=1:
$$




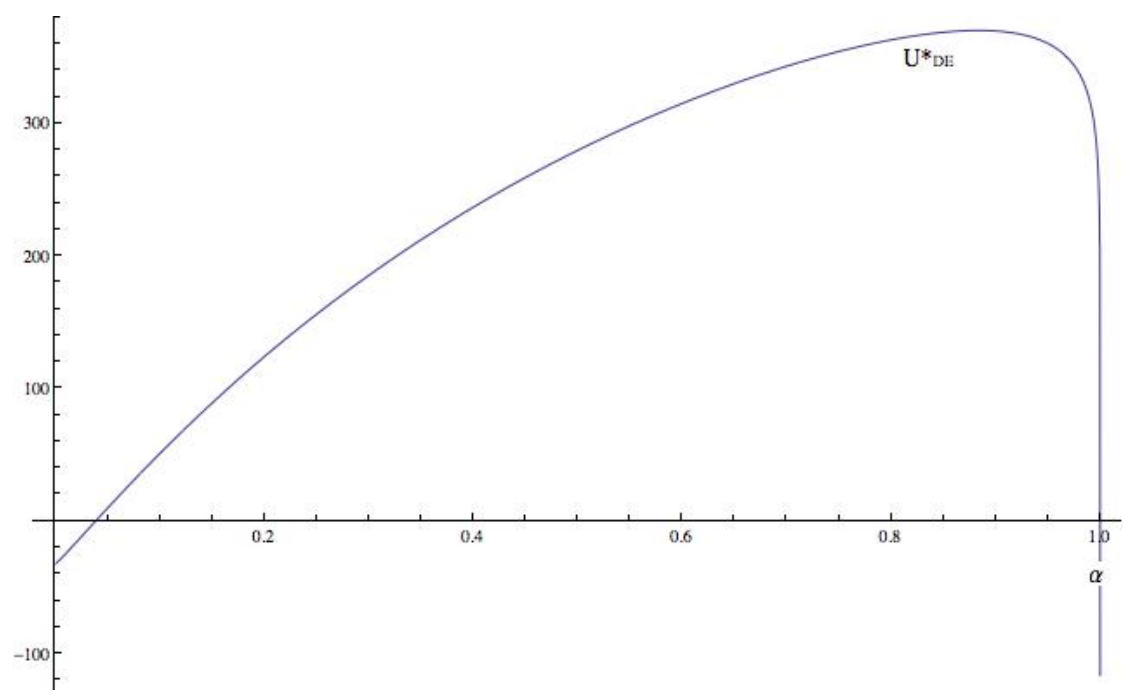

Figure 3.6: $\eta=1$ : The welfare function is an inverted-N shape

In the $\eta=5$ case the maximum welfare is attained for a value of $\alpha \simeq 0.4791$ which implies a Lerner index ${ }^{15}$ of 0.5208 , while in the $\eta=1$ case the maximum is for $\alpha \simeq 0.8836$ and corresponding Lerner index is 0.1164. Both of these indexes are in line with Hall's (1986) estimations, being in the range [0.048, 0.705].

Given the expression of the first derivative (3.2) it is difficult to find the turning points analytically. We can provide a solution of equation (3.2) in a neighborhood of $\alpha=1$, which corresponds to the value of $\alpha$ for which the welfare function attains a maximum. By rewriting the first derivative as

$$
\ln \alpha=\left[-\frac{L+\rho \eta}{\rho \eta(1+\alpha)^{2}}(1-\alpha)-1\right] \frac{1-\alpha}{2}
$$

and by setting $\alpha=1-\epsilon$, we obtain the following second order equation in $\epsilon^{16}$ :

$$
\rho \eta \epsilon^{2}-(L+5 \rho \eta) \epsilon+4 \rho \eta=0
$$

whose solutions are

$$
\epsilon_{1,2}=\frac{(L+5 \rho \eta) \pm \sqrt{(L+5 \rho \eta)^{2}-16 \rho^{2} \eta^{2}}}{2 \rho \eta}=\frac{(L+5 \rho \eta) \pm \sqrt{L^{2}+9 \rho \eta^{2}+10 L \rho \eta}}{2 \rho \eta} .
$$

\footnotetext{
${ }^{15}$ In this model the mark up, as defined as $\frac{\text { Price-Marginal Cost }}{\text { Marginal Cost }}$, is equal to $\frac{1}{\alpha}-1$. The Lerner index, which is a good approximation of the price-cost margin, $\frac{\text { Price-Marginal Cost }}{\text { Pricet }}$, is related to the mark up in the following way: Lerner Index $=\frac{\text { Mark Up }}{1+\text { Mark Up }}$.

${ }^{16}$ We recall that $\ln (1-\epsilon) \simeq-\epsilon$.
} 
Since

$$
\begin{gathered}
\epsilon_{1}=\frac{(L+5 \rho \eta)+\sqrt{(L+5 \rho \eta)^{2}-16 \rho^{2} \eta^{2}}}{2 \rho \eta}>1 \\
\epsilon_{2}=\frac{(L+5 \rho \eta)-\sqrt{(L+5 \rho \eta)^{2}-16 \rho^{2} \eta^{2}}}{2 \rho \eta}<1 .
\end{gathered}
$$

$\epsilon_{1}$ implies that $\alpha<0$, while with $\epsilon_{2}$ we get a solution for $\alpha$ in the interval $(0,1)$ which is given by:

$$
\alpha^{*}=\frac{-3 \rho \eta-L+\sqrt{(L+5 \rho \eta)^{2}-16 \rho^{2} \eta^{2}}}{2 \rho \eta} .
$$

This expression provides a good approximation of the value of $\alpha$ which maximizes welfare in the decentralized economy, if it is in a neighborhood of 1 . For example, in the $\eta=1$ case, the true value of $\alpha$ which maximizes welfare is 0.8836 and $\alpha^{*}=0.8954$.

\section{Conclusions}

In this paper we considered the model of horizontal differentiation reported in Barro and Salai-Martin (2004), Ch. 6, which is a simplified version of Romer's (1990) model and derived the closed form solutions for the level of the variables in the decentralized economy and in the social planner case. This enabled us to analyze consumers' welfare as a function of the parameter representing the degree of competition. As is well known in this model an increase in competition enhances the decentralized economy's growth rate because it causes a reallocation of human capital toward the R\&D sector, while the centralized economy's growth rate is independent from the competition parameter because the social planner eliminates the distortion due to the presence of monopolistic competition. As a result of welfare analysis, instead, we surprisingly find that, when the total labor force is greater than a certain level, the welfare function is an inverted-N shape in the decentralized economy and monotonically decreasing in the centralized economy. This suggests that there is another effect which interacts with market power: the degree of returns to specialization effect. 


\section{Appendix. Proof of Proposition 3}

Given the solution for the level of consumption:

$$
C(t)_{D E}=\alpha^{\frac{2 \alpha}{1-\alpha}}(1-\alpha) N(0)(L+\rho \eta) \exp \left[\frac{\alpha L-\rho \eta}{\eta(1+\alpha)} t\right]
$$

we can compute the representative consumer's welfare:

$$
\begin{aligned}
& U_{D E}^{*}= \\
&=\int_{0}^{+\infty} \ln \left\{\alpha^{\frac{2 \alpha}{1-\alpha}}(1-\alpha) N(0)(L+\rho \eta) \exp \left[\frac{\alpha L-\rho \eta}{\eta(1+\alpha)} t\right]\right\} \exp [-\rho t] d t \\
&= \\
& \frac{1}{\rho}\left\{\ln \left[\alpha^{\frac{2 \alpha}{1-\alpha}}(1-\alpha) N(0)(L+\rho \eta)\right]+\frac{1}{\rho} \frac{\alpha L-\rho \eta}{\eta(1+\alpha)}\right\} .
\end{aligned}
$$

Deriving it with respect to $\alpha$ gives

$$
\frac{\partial U_{D E}^{*}}{\partial \alpha}=\frac{1}{\rho}\left\{\frac{1}{1-\alpha}\left[\frac{2}{1-\alpha} \ln \alpha+1\right]+\frac{1}{\rho \eta} \frac{L+\rho \eta}{(1+\alpha)^{2}}\right\} .
$$

The sign of the curly bracket is ambiguous since $\ln \alpha<0$ for $\alpha \in(0,1)$. In order to study it we define

$$
f(\alpha) \equiv \frac{1}{1-\alpha}\left[\frac{2}{1-\alpha} \ln \alpha+1\right]+\frac{1}{\rho \eta} \frac{L+\rho \eta}{(1+\alpha)^{2}} .
$$

This function is continuous for $\alpha \in(0,1)$ and $\lim _{\alpha \rightarrow 0} f(\alpha)=\lim _{\alpha \rightarrow 1} f(\alpha)=-\infty$. However given the fact that it depends on the parameters of the model, $L, \eta$ and $\rho$, we are not able to say anything about the existence of some intersection with the $\alpha-\operatorname{axis}^{17}$ and, if they exist, how many they are. To this end, we define:

$$
g(\alpha) \equiv \frac{1}{1-\alpha}\left[\frac{2}{1-\alpha} \ln \alpha+1\right]
$$

and

$$
h(\alpha) \equiv \frac{1}{\rho \eta} \frac{L+\rho \eta}{(1+\alpha)^{2}} .
$$

The function $g(\alpha)$ is continuous in $(0,1)$, always negative, and concave. Moreover $\lim _{\alpha \rightarrow 0} g(\alpha)=$ $\lim _{\alpha \rightarrow 1} g(\alpha)=-\infty$. The function $h(\alpha)$ is continuous in $(0,1)$, always positive, decreasing and convex. Moreover $\lim _{\alpha \rightarrow 0} h(\alpha)=\frac{L+\rho \eta}{\rho \eta}$ and $\lim _{\alpha \rightarrow 1} h(\alpha)=\frac{L+\rho \eta}{4 \rho \eta}$ (see Figure 3.1 in the text).

In order to understand if there exist some values of $\alpha$ such that the sum of the two functions is zero we take the symmetric of $g(\alpha)$ with respect to the $\alpha-$ axis, $-g(\alpha)$. If $-g(\alpha)$ and $h(\alpha)$ cross somewhere, this means that there exists a value of $\alpha$ such that $f(\alpha)=0$. Three situations can arise, depending on the configuration of the parameters of the model $L, \eta$ and

\footnotetext{
${ }^{17}$ This would mean that the function $U_{D E}^{*}$ changes its slope.
} 
$\rho$ (see Figure 3.2 in the text):

Case 1: $h(\alpha)$ and $-g(\alpha)$ do not intersect, hence the relationship between welfare and $\alpha$ is monotonically decreasing;

Case 2: $h(\alpha)$ and $-g(\alpha)$ are tangent, hence the relationship between welfare and $\alpha$ is monotonically decreasing and it just shows a change of concavity;

Case 3: $h(\alpha)$ and $-g(\alpha)$ cross twice, hence the relationship between welfare and $\alpha$ is an inverted-N shape.

Given that $h(0)=\frac{L+\rho \eta}{\rho \eta}$ and $h(1)=\frac{L+\rho \eta}{4 \rho \eta}$, Case 3 is more likely to occur the greater is $L$ and the smaller are $\eta$ and $\rho^{18}$. To find a sufficient condition for Case 3 to occur we compute $h(\alpha)$ for the value of $\alpha$ such that $-g(\alpha)$ attains a minimum, $\alpha_{M I N} \equiv \min \left(-\frac{1}{1-\alpha}\left[\frac{2}{1-\alpha} \ln \alpha+1\right]\right) 19$. If $h\left(\alpha_{M I N}\right) \geq-g\left(\alpha_{M I N}\right)^{20}$, the two functions intersect twice. This happens for

$$
L \geq \underline{L}
$$

where $\underline{L}=\lambda \rho \eta, \lambda \equiv f\left(\min \left(-\frac{1}{1-\alpha}\left[\frac{2}{1-\alpha} \ln \alpha+1\right]\right)\right)^{21}$.

\footnotetext{
${ }^{18}$ We will talk about the role of these parameters in Section 3.2.2.

${ }^{19}$ This value is approximately equal to $\alpha_{M I N} \simeq 0.383012$.

${ }^{20}$ where $-g\left(\alpha_{M I N}\right) \simeq 3.42127$.

${ }^{21} \lambda \simeq 5.54394$.
} 


\section{References}

[1] Aghion, P., Bloom, N., Blundell, R., Griffith, R., Howitt, P. (2005), "Competition and innovation: an inverted-U relationship", The Quarterly Journal of Economics, 120, 701728 .

[2] Barro, R., Sala-i-Martin, X. (2004). Economic growth. New York, McGraw-Hill.

[3] Benassy, J-P. (1998), "Is there always too little research in endogenous growth with expanding product variety?", European Economic Review, 42, 61-69.

[4] Blundell, R., Griffith, R. and Van Reenen, J. (1999), "Market share, market value and innovation in a panel of british manufacturing firms", Review of Economic Studies, 66, 529-554.

[5] Bucci, A. (2003), "Market Power and Aggregate Economic Growth in Models with Endogenous Technological Change," Giornale degli Economisti e Annali di Economia, 62, 241-291.

[6] Bucci, A. (2005), "An Inverted-U Relationship between Product Market Competition and Growth in an Extended Romerian Model," Rivista di Politica Economica, SIPI Spa, 95, $177-206$.

[7] Geroski, P. (1995). Market structure, corporate performance and innovative activity. Oxford: Oxford University Press.

[8] Grossman, G. M., Helpman, E. (1991). Innovation and growth in the global economy. Cambridge, MA: MIT Press.

[9] Hall, R. E. (1986), "Market structure and macroeconomic fluctuations", Brookings Papers on Economic Activity, 2, 285-338.

[10] Onori, D. (2010), "Competition and growth: reinterpreting their relationship," CORE Discussion Papers 2010033, Université catholique de Louvain, Center for Operations Research and Econometrics (CORE)

[11] Romer, P. M. (1990), "Endogenous technical change", Journal of Political Economy, 98, 469-89. 